\section{Case Reports in Neurology}

\title{
Autologous Bone Marrow Transplantation for Polymyositis Combined with Myasthenia Gravis and Aplastic Anemia: A Case Report
}

\author{
Sho Mitsumune ${ }^{a} \quad$ Yasuhiro Manabe $^{a} \quad$ Taijun Yunoki $^{a}$ Shyoichiro Kono \\ Kazutoshi Aoyama ${ }^{b}$ Yoko Shinno $^{c}$ Hisashi Narai ${ }^{a}$ Koji Abe ${ }^{d}$ \\ aDepartment of Neurology, National Hospital Organization Okayama Medical Center, \\ Okayama, Japan; bepartment of Hematology, National Hospital Organization Okayama \\ Medical Center, Okayama, Japan; ${ }^{c}$ Clinical Laboratory, National Hospital Organization \\ Okayama Medical Center, Okayama, Japan; ${ }^{d}$ Department of Neurology, Graduate School \\ of Medicine, Dentistry, and Pharmaceutical Sciences, Okayama University, \\ Okayama, Japan
}

\section{Keywords}

Polymyositis · Myasthenia gravis · Aplastic anemia $\cdot$ Bone marrow transplantation

\begin{abstract}
This is the first report about a patient with concomitant polymyositis (PM), myasthenia gravis $(\mathrm{MG})$, and aplastic anemia (AA). A 54-year-old male developed myalgia and muscle weakness, which gradually progressed over 2 months. He was persistently affected by MG and AA. Brachium magnetic resonance imaging showed increased signal intensity in the left triceps and deltoid muscles on short tau inversion recovery images. A muscle biopsy examination revealed perifascicular atrophication and inflammatory myopathy. We diagnosed the patient with PM combined with MG and AA. He was successfully treated with an autologous bone marrow transplantation (BMT). The present case suggests that BMT is a therapeutic option for PM, MG, and AA.




\section{Introduction}

Polymyositis (PM) is an idiopathic type of inflammatory myopathy which causes symmetrical, proximal muscle weakness. Myasthenia gravis (MG) is another autoimmune neuromuscular disease which leads to varying degrees of skeletal muscle weakness. Patients with myositis may also have other autoimmune diseases, such as systemic lupus erythematosus, systemic sclerosis, Sjögren syndrome, or MG [1]. Aplastic anemia (AA) is a syndrome involving bone marrow failure characterized by peripheral pancytopenia and marrow hypoplasia, and it can occur concomitantly with MG [2]. Thymoma is associated with autoimmune disorders, including MG, PM, hypogammaglobulinemia, agranulocytosis, pure red cell aplasia, and AA [2]. It is important to detect concomitant conditions as they can influence the optimal management strategy. However, no cases of concomitant PM, MG, and AA have been reported previously. Here, we report a case in whom autologous bone marrow transplantation (BMT) for PM combined with MG and AA was performed.

\section{Case Report}

A 54-year-old male developed myalgia and muscle weakness which gradually progressed over 2 months. He had a history of MG from the age of 27 years. He had undergone resection of the thymus at the ages of 27 and 50 years because of malignant thymoma, followed by four courses of adjuvant chemotherapy. He had been treated with prednisolone $(10 \mathrm{mg}$ ) every other day and daily cyclosporine $(70 \mathrm{mg})$. He had also suffered from AA from the age of 50 years (since the adjuvant chemotherapy) and diabetes mellitus for 20 years. On admission, general physical examination obtained normal findings. Neurological examination showed that his cranial nerves were intact. He did not have dysarthria, ptosis, or dysphagia. Motor examination revealed strength scores of $3 / 5$ for the bilateral deltoid muscles and 4/5 for the bilateral arm extensor and flexor muscles, hip extensor and flexor muscles, and knee extensor and flexor muscles, as delineated by the Medical Research Council of Great Britain (MRC). Gowers' sign was present. All deep tendon reflexes were normal. The bilateral Babinski reflexes were absent. Laboratory studies revealed reductions in the patient's red blood cell count $\left(293 \times 10^{4} / \mu \mathrm{L}\right)$, hemoglobin level $(8.4 \mathrm{~g} / \mathrm{dL})$, and platelet count $\left(4.6 \times 10^{4} / \mu \mathrm{L}\right)$, a high anti-acetylcholine receptor antibody titer $(150 \mathrm{nmol} / \mathrm{L})$, and elevated creatine kinase (CK) $(2,536 \mathrm{U} / \mathrm{L}$; normal: $59-248 \mathrm{U} / \mathrm{L})$ and myoglobin $(2,722 \mathrm{ng} / \mathrm{mL}$; normal: 0-70 ng/mL) levels. Tests for anti-Jo-1 and anti-aminoacyl-tRNA synthetase antibodies produced negative results. Brachium magnetic resonance imaging showed increased signal intensity in the left triceps and deltoid muscles on T2-weighted imaging and short tau inversion recovery imaging (Fig. 1a, b, arrows). Muscle biopsy examination of the left biceps brachii muscle revealed perifascicular atrophication and inflammatory myopathy (Fig. 1c, d). We diagnosed the patient with PM combined with MG and AA. He was treated with two courses of intravenous immunoglobulin therapy, prednisolone $(20 \mathrm{mg})$ every other day, daily pyridostigmine $(90 \mathrm{mg})$, and daily cyclophosphamide $(40 \mathrm{mg})$. At 1 month after the diagnosis of PM, the patient's CK level had decreased to $705 \mathrm{U} / \mathrm{L}$. Motor examination revealed strength scores of $4 / 5$ for the right deltoid muscle, $3 / 5$ for the left deltoid muscle, $5 / 5$ for the right biceps brachii muscle, $4 / 5$ for the left biceps brachii muscle, $4 / 5$ for the bilateral triceps muscles, $4 / 5$ for the bilateral hip extensor and flexor muscles, and $4 / 5$ for the bilateral knee extensor and flexor muscles, as delineated by the MRC. The patient received a hu- 
man leukocyte antigen-mismatched BMT for AA (stage IV) 3 months after the diagnosis of PM. After the BMT, his CK level decreased to $38 \mathrm{U} / \mathrm{L}$, and his muscle strength improved.

\section{Discussion}

We report a case of concomitant PM, MG, and AA. Patients with PM can also develop other autoimmune diseases, such as systemic lupus erythematosus, systemic sclerosis, Sjögren syndrome, or MG [1]. AA can also occur concomitantly with MG [2]. However, there have not been any reported cases of concomitant PM, MG, and AA. Thymoma is associated with autoimmune disorders, including MG, PM, hypogammaglobulinemia, agranulocytosis, pure red cell aplasia, and AA [2]. The thymus is an important organ in which T cells develop and mature with the help of epithelial cells. Thymomas usually contain various proportions of epithelial cells and $\mathrm{T}$ cells. Cytotoxic $\mathrm{T}$ cells play a major role in destroying abnormal stem cells in the bone marrow and damaged muscle tissue [3]. A common thymoma-mediated mechanism might have been responsible for the concomitant PM, MG, and AA seen in our case.

BMT is the standard treatment for AA and can be used to treat autoimmune diseases, such as systemic lupus erythematosus, rheumatoid arthritis, immune thrombocytopenic purpura, and chronic glomerulonephritis [4]. Although MG and PM are both rare manifestations of immune dysregulation in chronic graft-versus-host disease, which can occur after BMT [5], the use of autologous hematopoietic stem cell transplants to treat MG or autologous peripheral blood stem cell transplants to treat PM has rarely been reported [6, 7]. The complete recovery of broad $\mathrm{T}$ cell immunity after hematopoietic stem cell transplantation or BMT might require generation of new naïve T cells from the thymus [8]. Douek et al. [9] suggested that the aged thymus contributes substantially to immune reconstitution early after hematopoietic stem cell transplantation, resulting in increased naïve $\mathrm{T}$ cell reconstitution. Immunological self-tolerance, focusing on naïve $\mathrm{T}$ cells, is newly reconstituted during this process. Our case indicates that BMT is a therapeutic option for PM, MG, and AA.

\section{Statement of Ethics}

The authors have no ethical conflicts to disclose.

\section{Disclosure Statement}

The authors state that they have no conflicts of interest and that they have no financial disclosure to make.

\section{References}

1 Paik JJ, Corse AM, Mammen AL. The co-existence of myasthenia gravis in patients with myositis: a case series. Semin Arthritis Rheum. 2014 Jun;43(6):792-6.

2 Gaglia A, Bobota A, Pectasides E, Kosmas C, Papaxoinis G, Pectasides D. Successful treatment with cyclosporine of thymoma-related aplastic anemia. Anticancer Res. 2007 Jul-Aug;27(4C):3025-8.

3 Nagasawa K. Thymoma-associated dermatomyositis and polymyositis. Intern Med. 1999 Feb;38(2):81-2.

4 Ikehara S. Bone marrow transplantation for autoimmune diseases. Acta Haematol. 1998;99(3):116-32. 


\section{Case Reports in Neurology}

5 Tse S, Saunders EF, Silverman E, Vajsar J, Becker L, Meaney B. Myasthenia gravis and polymyositis as manifestations of chronic graft-versus-host-disease. Bone Marrow Transplant. 1999 Feb;23(4):397-9.

6 Bryant A, Atkins H, Pringle CE, Allan D, Anstee G, Bence-Bruckler I et al. Myasthenia gravis treated with autologous hematopoietic stem cell transplantation. JAMA Neurol. 2016 Jun;73(6):652-8.

7 Baron F, Ribbens C, Kaye O, Fillet G, Malaise M, Beguin Y. Effective treatment of Jo-1-associated polymyositis with T-cell-depleted autologous peripheral blood stem cell transplantation. Br J Haematol. 2000 Aug;110(2): 339-42.

8 Heitger A, Neu N, Kern H, Panzer-Grümayer ER, Greinix H, Nachbaur D et al. Essential role of the thymus to reconstitute naive (CD45RA+) T-helper cells after human allogeneic bone marrow transplantation. Blood. 1997;90:850-9.

9 Douek DC, Vescio RA, Betts MR, Brenchiey JM, Hill BJ, Zhang L, Berenson JR, Collins RH, Koup RA. Assessment of thymic output in adults after haematopoietic stem-cell transplantation and prediction of T-cell reconstitution. Lancet. 2000;355:1875-81.
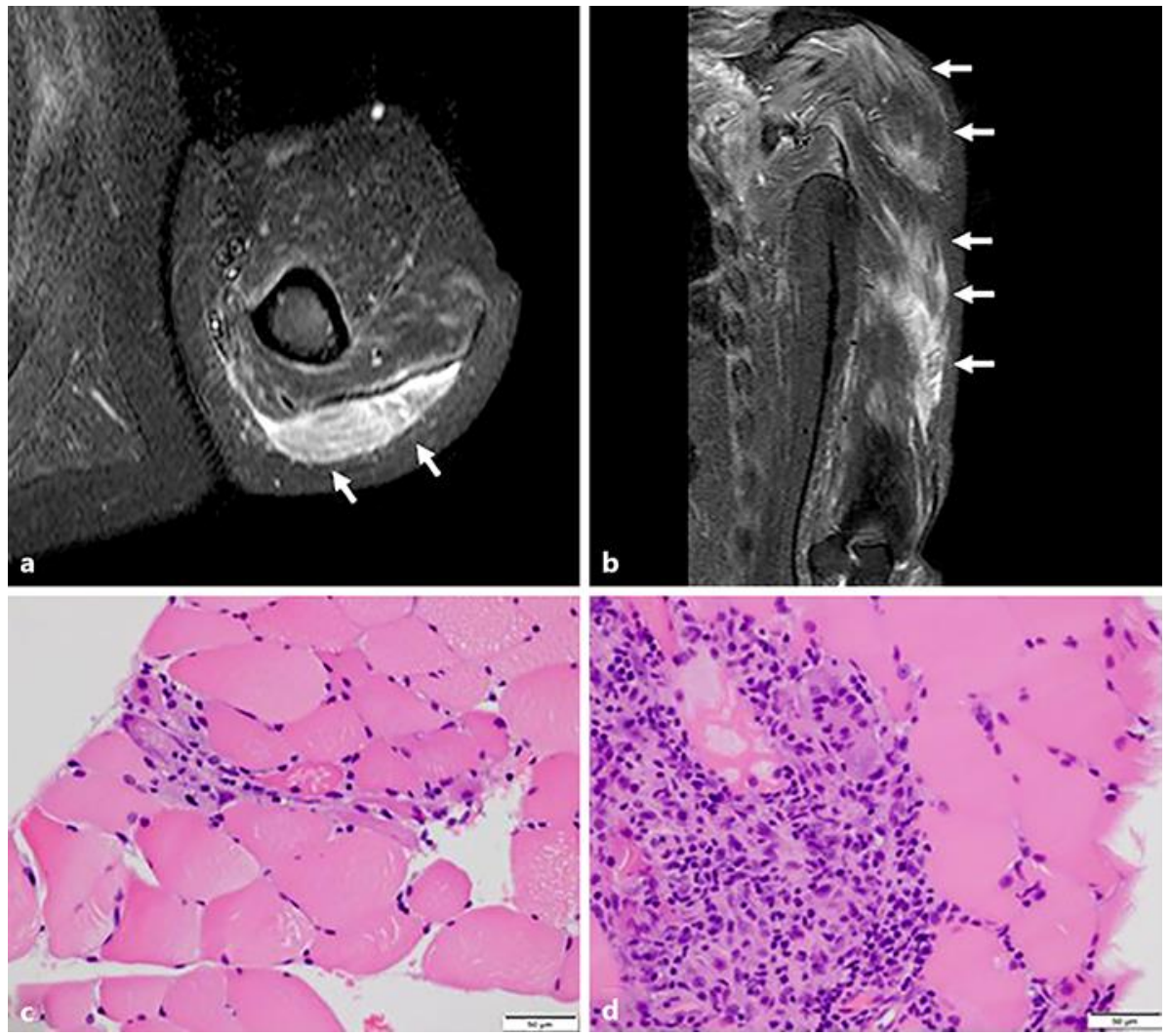

Fig. 1. a, b Brachium magnetic resonance imaging showed increased signal intensity in the left triceps and deltoid muscles on short tau inversion recovery imaging (arrows). c, $\mathbf{d}$ Muscle biopsy examination of the left biceps brachii muscle revealed perifascicular atrophication and inflammatory myopathy (hematoxylin and eosin staining). 